\title{
Household-Specific Physical Activity Levels and Energy Intakes According to the Presence of Metabolic Syndrome in Korean Young Adults: Korean National Health and Nutrition Examination Survey 2016-2018
}

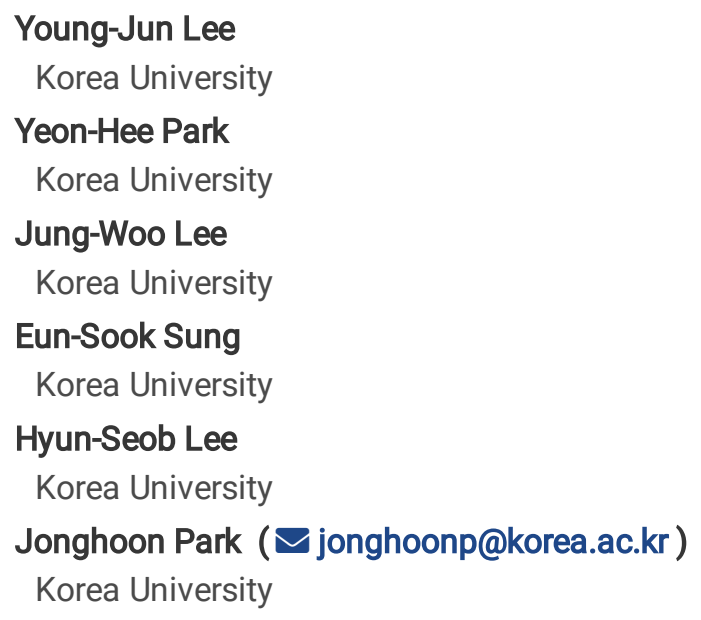

\section{Research Article}

Keywords: energy intake, metabolic syndrome, physical activity, single-person household

Posted Date: October 12th, 2021

DOI: https://doi.org/10.21203/rs.3.rs-923728/v1

License: @ (i) This work is licensed under a Creative Commons Attribution 4.0 International License. Read Full License

Version of Record: A version of this preprint was published at BMC Public Health on March 10th, 2022. See the published version at https://doi.org/10.1186/s12889-022-12852-3. 


\section{Abstract}

Background: Participation in exercise, and dietary and nutritional intakes have an impact on the risk and prevalence of metabolic syndrome (MetS), but these effects may differ according to whether a person lives alone or in a multi-person household. We analyzed differences in physical activity (PA) levels and energy intake according to household-type and MetS presence among young adults, to investigate the relationships among these factors.

Methods: Data of 3,974 young adults (aged $>19$ years and $<40$ years) were obtained from the Korean National Health and Nutrition Examination Survey (2016-2018). We analyzed PA levels (occupational and recreational PA, and place movement) and energy intake (total, carbohydrate, protein, and fat).

Results: Logistic regression data showed that low PA levels and higher energy intake were associated with MetS incidence and its components in young adults, after adjusting for body mass index, smoking, household-type, and sex. Overall, there was no significant difference in PA level between the MetS and non-MetS group. The total energy intake was higher in the MetS than in the non-MetS group $(p<0.05)$. These results were similar to those found in multi-person households. In single-person households, the MetS group had significantly lower PA levels $(p<0.01)$ and total energy intake $(p<0.05)$ than the non-MetS group.

Conclusions: We found significant association among low PA levels, high energy intake, and MetS components in young Korean adults, but with patterns differing according to household type. Energy intake was higher in young adults with than those without MetS, who lived in multi-person households, while young adults with MetS who lived alone had lower PA levels and lower energy intake than those without MetS. These findings highlight the need for different approaches of implementing PA and nutrition strategies according to the type of household in order to prevent MetS.

\section{Introduction}

The single-person household is the fastest growing type of household in many regions of the world, due to changes in institutional arrangements, demographic behaviors, and labor migration in the past few decades ${ }^{1}$. Not only widowed people, but also many young adults who have never been married, now live alone. The family structure in Korea has changed from the traditional large extended family to the nuclear family, due to industrialization and urbanization, and recently, the number of single-person households living alone in Korea has increased rapidly ${ }^{2}$. According to the National Statistical Office, the percentage of singleperson households has increased from $15.5 \%$ in 2000 , to $29.3 \%$ in 2018 , and is estimated to reach $34.3 \%$ by $2035^{3}$.

According to the Korean National Health and Nutrition Examination Survey (KNHANES), the incidence of metabolic syndrome (MetS) has increased by $0.6 \%$ every 10 years since $1998^{4}$. Additionally, recent studies in Korea have found that single-person households are more susceptible than multi-person households to insufficient physical activity (PA) and unhealthy eating practices

5. The amount of PA has decreased, with a decrease in moderate PA and walking, without an increase in high-intensity PA ${ }^{6,7}$. Low levels of PA, in turn, contribute to the increase in MetS incidence.

In conjunction with social changes in Korea, the dietary behavior of Koreans has also changed rapidly. With increased aging of the Korean population and increasing numbers of single-person households, eating alone has become a social concern. Some previous studies reported that eating alone was associated with various health problems. Eating alone was related to a reduced calorie intake and a less-varied diet ${ }^{8,9}$, and it could be a direct risk factor for Mets ${ }^{10}$.

The level of PA, the degree of participation in exercise, and dietary and nutritional intakes have an impact on the risk for and prevalence of MetS. In a recent comparative analysis, among 66,211 older individuals (aged 60 years or more) in Korea, singleperson households are considered to have worse overall health behaviors, such as exercise behaviors and nutritional behaviors, than multi-person households ${ }^{11}$. According to KNHANES data (2013-2015) of 2,903 subjects $\geq$ aged 65 years, single-person households had worse nutrient intake overall, and had an increased prevalence of MetS ${ }^{12}$.

Research on housing and MetS of young people, particularly young single-person households, has been scant internationally. Moreover, there have been few studies about household type-specific PA and energy intake in young adults according to the presence of MetS. Thus, it is important to examine the relationship between these factors in a large population, and even 
adequately-sized cohort studies evaluating PA levels and energy intakes according to household and the presence of MetS are not appropriate, because these factors vary according to young adults' lifestyles and household types. Therefore, this study aimed to analyze the differences in PA levels and energy intake by household type and the presence of MetS in a young adult Korean population, based on data from the 7th Korea National Health and Nutrition Examination Survey (2016-2018).

\section{Materials And Methods}

\subsection{Sample and Design}

This study used cross-sectional data from the Korea National Health and Nutrition Examination Survey (KNHANES) from 2016 to 2018, which was conducted by the Korea Centers for Disease Control and Prevention (KCDC). The details of the study design and data resource profiles followed the methods described in the Guidelines for Use of the KNHANES Raw Data and the Final Report of the sampling frame ${ }^{13}$. The KNHANES consists of a health interview survey, a nutrition survey, and a health examination, and is conducted according to the Declaration of Helsinki. This survey was approved by the Institutional Review Board of the Korea Centers for Disease Control and Prevention (reference number: 2018-01-03-P-A). All participants in the survey signed an informed consent form.

Between 2016 and 2018, 24,269 individuals completed the health interview survey, nutrition survey, and health examination. Among them, 19,031 people aged under 20 years or more than 40 years were excluded, leaving 5,238 people between the ages of 20 years and 39 years. Participants previously diagnosed with cancer (gastric, liver, colon, breast, cervical, lung, thyroid, and other cancers), and those with missing data (anthropometric, health examination, and PA data) were excluded (Figure 1). In total, 3,974 young people were finally included in this study.

\subsection{Measures}

The presence or absence of MetS was determined using measurements of waist circumference, blood pressure, fasting blood glucose levels, triglyceride levels, and HDL-C levels. PA variables were evaluated using the Global Physical Activity Questionnaire (GPAQ), and PA was expressed in metabolic equivalents (MET)-minutes/week. Nutrient intake was also analyzed. The analyzed characteristics of the participants according to household are shown in Table 1.

Table 1

Descriptive characteristics of the participants

\begin{tabular}{|c|c|c|c|c|c|c|c|c|c|}
\hline & \multicolumn{3}{|c|}{ Total $(n=3,974)$} & \multicolumn{3}{|c|}{ Multi-person Households $(n=3,619)$} & \multicolumn{3}{|c|}{ Single-person Households $(n=355)$} \\
\hline & $\begin{array}{l}\text { Non-MetS } \\
(n=3,477)\end{array}$ & $\begin{array}{l}\text { MetS } \\
(n=497)\end{array}$ & $p$-Value & $\begin{array}{l}\text { Non-MetS } \\
(n=3,169)\end{array}$ & $\begin{array}{l}\text { MetS } \\
(n=450)\end{array}$ & $p$-Value & $\begin{array}{l}\text { Non-MetS } \\
(n=308)\end{array}$ & $\begin{array}{l}\text { MetS } \\
(n=47)\end{array}$ & $p$-Value \\
\hline $\begin{array}{l}\text { Age } \\
\text { (years) }\end{array}$ & $29.6 \pm 0.2$ & $32.5 \pm 0.3$ & 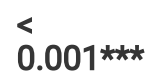 & $29.7 \pm 0.2$ & $32.7 \pm 0.3$ & <. $001 * \star *$ & $28.7 \pm 0.3$ & $31.3 \pm 0.9$ & $0.008 * *$ \\
\hline $\begin{array}{l}\text { Height } \\
\text { (cm) }\end{array}$ & $167.9 \pm 0.2$ & $172.3 \pm 0.4$ & $0.001 * * *$ & $167.7 \pm 0.2$ & $172.1 \pm 0.4$ & $<.001 * \star *$ & $170.0 \pm 0.4$ & $173.9 \pm 1.0$ & $\hat{0.001 * \star *}$ \\
\hline $\begin{array}{l}\text { Body } \\
\text { weight } \\
\text { (kg) }\end{array}$ & $64.9 \pm 0.3$ & $86.4 \pm 0.8$ & $<.001 * * *$ & $64.6 \pm 0.3$ & $85.7 \pm 0.9$ & $\hat{0.001 * * *}$ & $66.8 \pm 0.8$ & $91.9 \pm 1.7$ & <.001*** \\
\hline $\begin{array}{l}\text { BMI } \\
\left(\mathrm{kg} / \mathrm{m}^{2}\right)\end{array}$ & $22.9 \pm 0.1$ & $29.0 \pm 0.2$ & <. $001 * * *$ & $22.9 \pm 0.1$ & $28.8 \pm 0.3$ & $\hat{0.001 * * *}$ & $23.0 \pm 0.3$ & $30.3 \pm 0.5$ & $<.001$ *** \\
\hline $\begin{array}{l}\text { Alcohol } \\
(\%)\end{array}$ & 67.7 & 72.3 & 0.105 & 66.5 & 71.1 & 0.131 & 77.7 & 82.2 & 0.469 \\
\hline $\begin{array}{l}\text { Smoking } \\
(\%)\end{array}$ & 21.8 & 39.5 & <. $0.001 * * *$ & 20.5 & 39.6 & <. $001 * * \star$ & 32.9 & 38.9 & 0.516 \\
\hline
\end{tabular}




\subsection{Metabolic Syndrome}

The diagnosis of MetS was based on the new harmonized guidelines of the National Cholesterol Education Program-Adult Treatment Panel III and the American Heart Association and the National Heart Lung and Blood Institutes ${ }^{14,15}$. For waist circumference, we followed the criteria suggested by the Korean Society for the Study of Obesity ${ }^{16}$. If three or more of the following five criteria were met, participants were classified as having MetS: waist circumference $>90 \mathrm{~cm}$ (men) or $>85 \mathrm{~cm}$ (women); systolic blood pressure $>130 \mathrm{mmHg}$ or diastolic blood pressure $>85 \mathrm{mmHg}$; fasting triglyceride (TG) levels $>150 \mathrm{mg} / \mathrm{dL}$; fasting HDL-C levels $<40 \mathrm{mg} / \mathrm{dL}$ (men) or $<50 \mathrm{mg} / \mathrm{dL}$ (women); and fasting glucose levels $>100 \mathrm{mg} / \mathrm{dL}$.

\subsection{Physical Activity}

The GPAQ comprises 16 questions grouped to capture PA undertaken in different behavioral domains: work, transport, and discretionary (also known as leisure or recreational) activities. It analyzes five domains of PA: vigorous-intensity work, moderateintensity work, place movement, vigorous-intensity recreation, and moderate-intensity recreation. The World Health Organization (WHO) GPAQ analysis guidelines were used to analyze GPAQ data ${ }^{17}$. We estimated that, compared to while sitting quietly, a person's caloric consumption was four times higher when they were being moderately active and eight times higher when they were being vigorously active. Therefore, when calculating the total energy consumption of an individual using GPAQ data, four METs were allocated to the time spent in moderate activity and eight METs to the time spent in vigorous activity, and the details are as follows:

- Vigorous intensity activity: occupational $(\mathrm{MET})=8.0 \times$ vigorous intensity physical activity $($ day/week $) \times 1$-day vigorous intensity physical activity (minutes/day)

- Moderate intensity activity: occupational $(M E T)=4.0 \times$ moderate intensity physical activity (day/week) $\times 1$-day moderate intensity physical activity (minutes/day)

- Vigorous intensity activity: recreational $(M E T)=8.0 \times$ vigorous intensity physical activity (day/week) $\times 1$-day vigorous intensity physical activity (minutes/day)

- Moderate intensity activity: recreational $(M E T)=4.0 \times$ moderate intensity physical activity (day/week) $\times 1$-day moderate intensity physical activity (minutes/day)

- Place movement $(\mathrm{MET})=4.0 \times$ place movement physical activity (day/week) $\times 1$-day place movement physical activity

- Total Physical Activity (MET) = vigorous intensity activity: occupational + moderate intensity activity: occupational + vigorous intensity activity: recreational + moderate intensity activity: recreational + place movement.

PA levels were classified into four groups: inactive (0-249 MET min/week), somewhat active (250-499 MET min/week), active (500-999 MET min/week), and very active (>1000 MET min/week). These cut-off points are based on their equivalence to the following PA thresholds: 250 MET min/week represents an energy expenditure dose equivalent to half of threshold; 500 MET $\mathrm{min} /$ week is equivalent to the minimal threshold; and $1000 \mathrm{MET}$ min/week is equivalent to twice the minimal threshold.

\subsection{Energy Intake}

The nutrition survey of the KNHANES consisted of a survey of dietary habits, 1-day 24-hour recall, and food frequency questionnaire administration. The nutrition survey data were collected by trained dietitians in the homes of the participants 1 week after the health interview and health examination. The daily energy intake was calculated using the Korean Foods and Nutrients Database of the Rural Development Administration. The following items were included in the analyses: total energy intake, carbohydrate intake, protein intake, and fat intake. Energy intake was categorized by dividing the ratio with estimated energy requirement (EER). Values less than 20\% EER (<0.8) were considered as lower intake, whereas values above 1.2 as higher intake.

\subsection{Statistical Analysis}

Continuous variables were presented as means and standard errors. Normality of the distribution of all outcome-variable data was verified using the Kolmogorov-Smirnov test. An independent $t$-test was used to analyze risk factors for MetS, as well as PA levels and energy intakes between the non-MetS and MetS group. Two-way analyses of variance (ANOVA) were used to analyze the differences in risk factors for MetS, PA levels, energy intakes between participants with and without MetS, and between singleperson and multi-person households. Partial eta-squared (n2) values were calculated to represent effect sizes. If a significant 
interaction effect was found by two-way ANOVA, a Bonferroni post-hoc test was used to compare the household-specificity of dependent variables in each group (with and without MetS) separately. Moreover, the relationships between PA levels or energy intake and MetS were determined using logistic regression analysis after controlling for covariates. Logistic regression analysis findings were presented as odds ratios (ORs) and their associated $95 \%$ confidence intervals (Cls). Statistical analyses were performed using SPSS version 25.0 for Windows (IBM Corp., Armonk, NY, USA). The level of significance was set at 0.05.

\section{Results}

The average values of PA levels and energy intake factors are shown in Table 2 and the ORs for MetS and MetS components according to PA levels and energy intake are presented in Table 3. The ORs were adjusted for body mass index, smoking, household-type, and sex in model 2. We found that an "Active" PA level was associated with low HDL-C levels $(O \mathrm{R}=0.76,95 \% \mathrm{Cl}=$ $0.61-0.93)$. A PA level of "Very active" was associated with a lower MetS incidence $(\mathrm{OR}=0.55,95 \% \mathrm{Cl}=0.39-0.77)$, larger waist circumference $(O R=0.64,95 \% \mathrm{Cl}=0.43-0.93)$, high triglyceride $(T G)$ levels $(O R=0.69,95 \% \mathrm{Cl}=0.56-0.87)$, and low levels of highdensity lipoprotein $\mathrm{C}(\mathrm{HDL}-\mathrm{C})(\mathrm{OR}=0.72,95 \% \mathrm{Cl}=0.59-0.88)$. We also found that "Moderate energy intake" levels were associated with a larger waist circumference $(O R=1.46,95 \% \mathrm{Cl}=1.05-2.04)$ and high glucose levels $(\mathrm{OR}=1.23,95 \% \mathrm{Cl}=1.02-1.63)$. "Higher energy intake" levels were associated with the high TG levels $(\mathrm{OR}=1.33,95 \% \mathrm{Cl}=1.03-1.70)$, low $\mathrm{HDL}-\mathrm{C}$ levels $(\mathrm{OR}=0.77,95 \% \mathrm{Cl}=$ $0.61-0.97)$, and high glucose levels $(\mathrm{OR}=1.39,95 \% \mathrm{Cl}=1.01-1.92)$.

Table 2

Classification of physical activity levels and energy intake

\begin{tabular}{|c|c|c|c|c|c|c|}
\hline Factors & Total & $\mathrm{n}$ & $\begin{array}{l}\text { Multi-person } \\
\text { Households }\end{array}$ & $\mathrm{n}$ & $\begin{array}{l}\text { Single-person } \\
\text { Households }\end{array}$ & $\mathrm{n}$ \\
\hline Physical activity factors & \multicolumn{6}{|c|}{ MET min/Week (Mean $\pm S E)$} \\
\hline Inactive & $39.6 \pm 2.5$ & 1354 & $40.0 \pm 2.6$ & 1255 & $35.4 \pm 7.6$ & 99 \\
\hline Somewhat active & $406.9 \pm 3.2$ & 464 & $406.1 \pm 3.3$ & 428 & $415.9 \pm 11.3$ & 36 \\
\hline Active & $750.7 \pm 5.3$ & 834 & $752.5 \pm 5.8$ & 746 & $739.2 \pm 12.5$ & 88 \\
\hline Very active & $2775.1 \pm 100.2$ & 1322 & $2754.4 \pm 97.8$ & 1190 & $2944.4 \pm 345.2$ & 132 \\
\hline Energy intake factors & \multicolumn{6}{|c|}{ Energy intake/EER (Mean \pm SE) } \\
\hline $\begin{array}{l}\text { Lower energy intake } \\
\text { (energy intake/EER<0.8) }\end{array}$ & $0.6 \pm 0.0$ & 2067 & $0.6 \pm 0.0$ & 1884 & $0.5 \pm 0.0$ & 183 \\
\hline $\begin{array}{l}\text { Moderate energy intake (0.8 } \leq \text { energy } \\
\text { intake/EER } \leq 1.2)\end{array}$ & $1.0 \pm 0.0$ & 1288 & $1.0 \pm 0.0$ & 1163 & $1.0 \pm 0.0$ & 125 \\
\hline $\begin{array}{l}\text { Higher energy intake } \\
\text { (energy intake/EER>1.2) }\end{array}$ & $1.5 \pm 0.0$ & 619 & $1.5 \pm 0.0$ & 572 & $1.4 \pm 0.0$ & 47 \\
\hline
\end{tabular}


Table 3

Odds ratio (95\% Cl) for MetS and MetS components according to physical activity levels and energy intake

\begin{tabular}{|c|c|c|c|c|c|c|}
\hline Factors & MetS & $\begin{array}{l}\text { Large Waist } \\
\text { Circumference }\end{array}$ & $\begin{array}{l}\text { High } \\
\text { Triglycerides }\end{array}$ & Low HDL- & $\begin{array}{l}\text { High } \\
\text { Blood } \\
\text { Pressure }\end{array}$ & $\begin{array}{l}\text { High } \\
\text { Glucose }\end{array}$ \\
\hline \multicolumn{7}{|l|}{ Model 1 1) } \\
\hline \multicolumn{7}{|l|}{ Physical activity factors } \\
\hline \multirow[t]{2}{*}{ Inactive $(n=1354)$} & 1.00 & 1.00 & 1.00 & 1.00 & 1.00 & 1.00 \\
\hline & (reference) & (reference) & (reference) & (reference) & (reference) & (reference) \\
\hline \multirow[t]{2}{*}{ Somewhat active $(n=464)$} & 0.79 & 0.89 & 0.69 & 0.80 & 0.71 & 0.84 \\
\hline & $\begin{array}{l}(0.54- \\
1.16)\end{array}$ & $(0.64-1.23)$ & $(0.51-0.94) *$ & $\begin{array}{l}(0.59- \\
1.09)\end{array}$ & $(0.48-1.05)$ & $\begin{array}{l}(0.57- \\
1.24)\end{array}$ \\
\hline \multirow[t]{2}{*}{ Active $(n=834)$} & 0.86 & 0.927 & 0.78 & 0.77 & 1.10 & 0.99 \\
\hline & $\begin{array}{l}(0.64- \\
1.16)\end{array}$ & $(0.711-1.207)$ & $(0.62-0.97) *$ & $\begin{array}{l}(0.63- \\
0.94) *\end{array}$ & $(0.83-1.46)$ & $\begin{array}{l}(0.72- \\
1.35)\end{array}$ \\
\hline \multirow[t]{2}{*}{ Very active $(n=1322)$} & 0.84 & 1.02 & 0.88 & 0.74 & 1.15 & 0.96 \\
\hline & $\begin{array}{l}(0.63- \\
1.12)\end{array}$ & $(0.81-1.29)$ & $(0.72-1.08)$ & $\begin{array}{l}(0.61- \\
0.89)^{* *}\end{array}$ & $(0.89-1.49)$ & $\begin{array}{l}(0.75- \\
1.22)\end{array}$ \\
\hline \multicolumn{7}{|l|}{ Energy intake factors } \\
\hline Lower energy intake & 1.00 & 1.00 & 1.00 & 1.00 & 1.00 & 1.00 \\
\hline (energy intake/EER<0.8) & (reference) & (reference) & (reference) & (reference) & (reference) & (reference) \\
\hline \multirow{2}{*}{$\begin{array}{l}\text { Moderate energy intake } \\
(0.8 \leq \text { energy intake/EER } \leq 1.2)\end{array}$} & 0.85 & 0.77 & 0.92 & 0.90 & 0.90 & 1.07 \\
\hline & $\begin{array}{l}(0.66- \\
1.10)\end{array}$ & $(0.63-0.93) * *$ & $(0.74-1.14)$ & $\begin{array}{l}(0.76- \\
1.07)\end{array}$ & $(0.73-1.12)$ & $\begin{array}{l}(0.84- \\
1.36)\end{array}$ \\
\hline Higher energy intake & 0.84 & 0.77 & 1.16 & 0.68 & 0.86 & 1.16 \\
\hline (energy intake/EER>1.2) & $\begin{array}{l}(0.62- \\
1.13)\end{array}$ & $(0.58-1.02)$ & $(0.93-1.46)$ & $\begin{array}{l}(0.54- \\
0.85)^{\star \star}\end{array}$ & $(0.64-1.15)$ & $\begin{array}{l}(0.86- \\
1.55)\end{array}$ \\
\hline \multicolumn{7}{|l|}{ Model 2 2) } \\
\hline \multicolumn{7}{|l|}{ Physical activity factors } \\
\hline \multirow[t]{2}{*}{ Inactive $(n=1354)$} & 1.00 & 1.00 & 1.00 & 1.00 & 1.00 & 1.00 \\
\hline & (reference) & (reference) & (reference) & (reference) & (reference) & (reference) \\
\hline \multirow[t]{2}{*}{ Somewhat active $(n=464)$} & 0.91 & 1.10 & 0.75 & 0.80 & 0.77 & 0.92 \\
\hline & $\begin{array}{l}(0.58- \\
1.41)\end{array}$ & $(0.68-1.80)$ & $(0.53-1.06)$ & $\begin{array}{l}(0.58- \\
1.11)\end{array}$ & $(0.51-1.18)$ & $\begin{array}{l}(0.62- \\
1.36)\end{array}$ \\
\hline \multirow[t]{2}{*}{ Active $(n=834)$} & 0.86 & 1.05 & 0.78 & 0.76 & 1.12 & 1.01 \\
\hline & $\begin{array}{l}(0.59- \\
1.24)\end{array}$ & $(0.65-1.70)$ & $(0.60-1.01)$ & $\begin{array}{l}(0.61- \\
0.93)^{\star \star}\end{array}$ & $(0.82-1.52)$ & $\begin{array}{l}(0.74- \\
1.39)\end{array}$ \\
\hline \multirow[t]{2}{*}{ Very active $(n=1322)$} & 0.55 & 0.64 & 0.69 & 0.72 & 0.90 & 0.81 \\
\hline & $\begin{array}{l}(0.39- \\
0.77) \star \star \star\end{array}$ & $(0.43-0.93) *$ & $\begin{array}{l}(0.56- \\
0.87)^{* *}\end{array}$ & $\begin{array}{l}(0.59 \\
0.88)^{* *}\end{array}$ & $(0.67-1.19)$ & $\begin{array}{l}\text { (0.63- } \\
1.05)\end{array}$ \\
\hline
\end{tabular}

Data presented as odds ratio (95\% confidence intervals $(\mathrm{Cls})$ ). OR = odds ratio, MetS = metabolic syndrome, $\mathrm{HDL}-\mathrm{C}=\mathrm{high}-$ density lipoprotein cholesterol, EER $=$ estimated energy requirements. * $p<0.05$ vs. reference, ${ }^{\star \star} p<0.01$ vs. reference, ${ }^{\star * \star} p<$ 0.001 vs. reference; ${ }^{1)}$ Model 1: crude. ${ }^{2}$ ) Model 2: adjusted for sex, smoking, household type, and body mass index. 


\begin{tabular}{|c|c|c|c|c|c|c|}
\hline Factors & MetS & $\begin{array}{l}\text { Large Waist } \\
\text { Circumference }\end{array}$ & $\begin{array}{l}\text { High } \\
\text { Triglycerides }\end{array}$ & $\begin{array}{l}\text { Low HDL- } \\
\text { C }\end{array}$ & $\begin{array}{l}\text { High } \\
\text { Blood } \\
\text { Pressure }\end{array}$ & $\begin{array}{l}\text { High } \\
\text { Glucose }\end{array}$ \\
\hline \multicolumn{7}{|l|}{ Energy intake factors } \\
\hline $\begin{array}{l}\text { Lower energy intake } \\
\text { (energy intake/EER<0.8) }\end{array}$ & $\begin{array}{l}1.00 \\
\text { (reference) }\end{array}$ & $\begin{array}{l}1.00 \\
\text { (reference) }\end{array}$ & $\begin{array}{l}1.00 \\
\text { (reference) }\end{array}$ & $\begin{array}{l}1.00 \\
\text { (reference) }\end{array}$ & $\begin{array}{l}1.00 \\
\text { (reference) }\end{array}$ & $\begin{array}{l}1.00 \\
\text { (reference) }\end{array}$ \\
\hline \multirow{2}{*}{$\begin{array}{l}\text { Moderate energy intake } \\
(0.8 \leq \text { energy intake/EER } \leq 1.2)\end{array}$} & 1.20 & 1.46 & 1.05 & 1.02 & 1.07 & 1.23 \\
\hline & $\begin{array}{l}(0.88- \\
1.64)\end{array}$ & $(1.05-2.04) *$ & $(0.83-1.32)$ & $\begin{array}{l}(0.85- \\
1.22)\end{array}$ & $(0.86-1.35)$ & $\begin{array}{l}(1.02- \\
1.63)^{*}\end{array}$ \\
\hline \multirow{2}{*}{$\begin{array}{l}\text { Higher energy intake } \\
\text { (energy intake/EER>1.2) }\end{array}$} & 1.19 & 1.58 & 1.33 & 0.77 & 1.01 & 1.39 \\
\hline & $\begin{array}{l}(0.81- \\
1.75)\end{array}$ & $(0.94-2.65)$ & $(1.03-1.70)^{*}$ & $\begin{array}{l}(0.61- \\
0.97)^{*}\end{array}$ & $(0.71-1.42)$ & $\begin{array}{l}(1.01- \\
1.92)^{*}\end{array}$ \\
\hline
\end{tabular}

The differences in variables considered risk factors for MetS, according to the presence or absence of MetS and according to households are presented in Table 4. In the total group, young individuals with MetS had significantly higher values of risk factors for MetS than did those in the non-MetS group (all variables, $p<0.001$ ). The interaction between the presence or absence of MetS and household type (single- or multi-person) was statistically significant for waist circumference, TG, HDL-C, diastolic blood pressure (DBP), fasting blood glucose (all $p<0.001$ ). The Bonferroni post-hoc test showed the following group differences: Individuals with MetS living in multi-person households had significantly larger waist circumference, TG, DBP, fasting blood glucose (all $p<0.001)$, but lower HDL-C $(p<0.001)$, than their counterparts without MetS. Individuals with MetS who lived alone had significantly larger waist circumference, TG, HDL-C, DBP, and fasting blood glucose than those without MetS (all $p<0.001$ ). Individuals with MetS living as single persons had statistically significantly greater waist circumference and higher DBP (both $p<$ 0.01) than individuals with MetS living in multi-person households. 
Table 4

Metabolic Syndrome Components

\begin{tabular}{|c|c|c|c|c|c|c|c|c|}
\hline \multirow[t]{2}{*}{ Factors } & \multirow[t]{2}{*}{ Group } & \multirow[t]{2}{*}{ Total } & \multicolumn{2}{|c|}{ Household type } & \multicolumn{4}{|c|}{ ANOVA } \\
\hline & & & Multiple & Single & F-valu & & $p$-Value $\left(\eta^{2}\right)$ & Power \\
\hline \multirow{3}{*}{$\begin{array}{l}\text { Waist circumference } \\
\text { (cm) }\end{array}$} & $\begin{array}{l}\text { Non- } \\
\text { MetS }\end{array}$ & $77.9 \pm 0.2$ & $77.8 \pm 0.2$ & $78.6 \pm 0.7$ & $\mathrm{H}$ & $62.542 * \star \star *$ & $0.000(0.006)$ & 1.000 \\
\hline & MetS & $95.63 \pm 0.5$ & $95.2 \pm 0.6 \dagger$ & $99.3 \pm 1.1 \dagger \neq$ & M & $1489.340 * \star \star$ & $0.000(0.068)$ & 1.000 \\
\hline & $p$-value & $<.001 * \star \star$ & & & $\mathrm{H} \times \mathrm{M}$ & $87.608 * \star \star$ & $0.000(0.008)$ & 1.000 \\
\hline \multirow{3}{*}{$\begin{array}{l}\text { TG } \\
(\mathrm{mg} / \mathrm{dL})\end{array}$} & \multirow{2}{*}{$\begin{array}{l}\text { Non- } \\
\text { MetS }\end{array}$} & $102.8 \pm 1.7$ & $102.2 \pm 1.9$ & $107.8 \pm 2.9$ & $\mathrm{H}$ & 2.390 & $0.092(0.000)$ & 0.485 \\
\hline & & $242.7 \pm 9.5$ & $243.5 \pm 9.9 \dagger$ & $236.1 \pm 24.6 \dagger$ & M & $748.808 * \star \star$ & $0.000(0.037)$ & 1.000 \\
\hline & $\begin{array}{l}\text { MetS } \\
p \text {-value }\end{array}$ & $<.001 * * *$ & & & $\mathrm{H} \times \mathrm{M}$ & $9.114 * \star \star$ & $0.000(0.001)$ & 0.976 \\
\hline \multirow{4}{*}{$\begin{array}{l}\text { HDL-C } \\
(\mathrm{mg} / \mathrm{dL})\end{array}$} & \multirow{2}{*}{$\begin{array}{l}\text { Non- } \\
\text { MetS }\end{array}$} & $54.9 \pm 0.3$ & $55.0 \pm 0.2$ & $53.8 \pm 0.9$ & $\mathrm{H}$ & 0.022 & $0.978(0.000)$ & 0.053 \\
\hline & & $40.9 \pm 0.4$ & $40.7 \pm 0.4 \dagger$ & $42.2 \pm 1.4 \dagger$ & M & $552.453^{\star \star \star}$ & $0.000(0.027)$ & 1.000 \\
\hline & MetS & $<.001 * * *$ & & & $\mathrm{H} \times \mathrm{M}$ & 22.619*** & $0.000(0.002)$ & 1.000 \\
\hline & $p$-value & & & & & & & \\
\hline \multirow{3}{*}{$\begin{array}{l}\text { SBP } \\
(\mathrm{mmHg})\end{array}$} & \multirow{2}{*}{$\begin{array}{l}\text { Non- } \\
\text { MetS }\end{array}$} & $109.5 \pm 0.3$ & $109.1 \pm 0.2$ & $112.4 \pm 0.9$ & $\mathrm{H}$ & 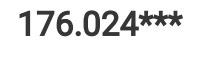 & $0.000(0.017)$ & 1.000 \\
\hline & & $123.0 \pm 0.6$ & $122.6 \pm 0.7$ & $125.9 \pm 2.3$ & M & 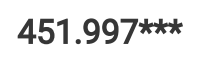 & $0.000(0.022)$ & 1.000 \\
\hline & $\begin{array}{l}\text { MetS } \\
p \text {-value }\end{array}$ & $\begin{array}{l}<.001 * \star * \\
0.01\end{array}$ & & & $\mathrm{H} \times \mathrm{M}$ & 0.485 & $0.485(0.616)$ & 0.130 \\
\hline \multirow{3}{*}{$\begin{array}{l}\text { DBP } \\
(\mathrm{mmHg})\end{array}$} & \multirow{3}{*}{$\begin{array}{l}\text { Non- } \\
\text { MetS } \\
\text { MetS } \\
p \text {-value }\end{array}$} & $72.6 \pm 0.2$ & $72.4 \pm 0.2$ & $74.8 \pm 0.6$ & $\mathrm{H}$ & $43.256 * \star *$ & $0.000(0.004)$ & 1.000 \\
\hline & & $85.1 \pm 0.5$ & $84.6 \pm 0.5 \dagger$ & $88.6 \pm 1.4 † \ddagger$ & M & 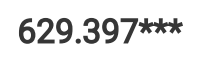 & $0.000(0.030)$ & 1.000 \\
\hline & & $<.001 * * *$ & & & $\mathrm{H} \times \mathrm{M}$ & $61.583 * \star \star$ & $0.000(0.006)$ & 1.000 \\
\hline \multirow{3}{*}{$\begin{array}{l}\text { Fasting glucose } \\
(\mathrm{mg} / \mathrm{dL})\end{array}$} & \multirow{3}{*}{$\begin{array}{l}\text { Non- } \\
\text { MetS } \\
\text { MetS } \\
p \text {-value }\end{array}$} & $90.3 \pm 0.3$ & $90.3 \pm 0.3$ & $90.4 \pm 0.5$ & $\mathrm{H}$ & $130.082 * \star \star$ & $0.000(0.013)$ & 1.000 \\
\hline & & $108.0 \pm 1.5$ & $108.5 \pm 1.6 \dagger$ & $104.2 \pm 2.7 \dagger$ & M & $359.141 \star \star \star$ & $0.000(0.018)$ & 1.000 \\
\hline & & $<.001 * * *$ & & & $\mathrm{H} \times \mathrm{M}$ & 7.636 *** & $0.000(0.001)$ & 0.948 \\
\hline \multicolumn{9}{|c|}{$\begin{array}{l}\text { Values are expressed as means standard errors; MetS, metabolic syndrome; TG, triglyceride; HDL-C, high-density lipoprotein } \\
\text { cholesterol; SBP, systolic blood pressure; DBP, diastolic blood pressure; HbA1c, glycated hemoglobin. Main effect }=\mathrm{H} \\
\text { (Household) and M (Metabolic syndrome), Interaction effect }=\mathrm{H} \times \mathrm{M} \text { (Household } \times \text { Metabolic syndrome), } p<0.05,{ }^{*} p<0.01 \text {, } \\
\star * \star p<0.001,+p<0.001 \text { compared with Non-MetS values, } \neq p<0.01 \text { compared with Multiple values. Two-way analysis of } \\
\text { variance following Bonferroni post-hoc test. }\end{array}$} \\
\hline
\end{tabular}

The differences in PA levels according to the presence or absence of MetS and according to household type are presented in Table 5. In the total group, there was no significant difference in PA levels between young individuals with and without MetS. For the PA aspects of "Occupational vigorous" ( $p<0.05)$, "Place movement" $(p<0.5)$, and "Total PA" $(p<0.01)$, there was a significant interaction between the presence or absence of MetS and household type. The Bonferroni post-hoc test showed the following group 
differences: Individuals with MetS living in multi-person households showed significantly higher "Occupational vigorous" PA ( $p<$ $0.05)$ than their counterparts without MetS. Those with MetS who lived alone demonstrated significantly lower "Place movement" and "Total" PA (all $p<0.01$ ) than their counterparts without MetS. There was no significant difference between those with MetS living in single-person households and those with MetS living in multi-person households.

Table 5

Levels of physical activity

\begin{tabular}{|c|c|c|c|c|c|c|c|c|}
\hline \multirow{2}{*}{$\begin{array}{l}\text { Physical } \\
\text { Activity } \\
\text { (MET } \\
\text { min/week) }\end{array}$} & \multirow[t]{2}{*}{ Group } & \multirow[t]{2}{*}{ Total } & \multicolumn{2}{|l|}{ Household type } & \multicolumn{4}{|c|}{ ANOVA } \\
\hline & & & Multiple & Single & F-valu & & $p$-Value $\left(n^{2}\right)$ & Power \\
\hline \multirow{3}{*}{$\begin{array}{l}\text { Occupational } \\
\text { vigorous }\end{array}$} & $\begin{array}{l}\text { Non- } \\
\text { MetS }\end{array}$ & $76.7 \pm 15.5$ & $65.1 \pm 12.8$ & $176.0 \pm 86.9$ & $\mathrm{H}$ & $3.765^{*}$ & $0.023(0.000)$ & 0.689 \\
\hline & \multirow{2}{*}{$\begin{array}{l}\text { MetS } \\
p \text {-value }\end{array}$} & $132.4 \pm 47.8$ & $148.5 \pm 53.5 \dagger$ & $1.7 \pm 1.7$ & $M$ & 0.836 & $0.361(0.000)$ & 0.150 \\
\hline & & 0.271 & & & $\mathrm{H} \times \mathrm{M}$ & $4.575^{\star}$ & $0.010(0.000)$ & 0.778 \\
\hline \multirow{3}{*}{$\begin{array}{l}\text { Occupational } \\
\text { moderate }\end{array}$} & \multirow{2}{*}{$\begin{array}{l}\text { Non- } \\
\text { MetS }\end{array}$} & $214.6 \pm 23.9$ & $214.0 \pm 25.0$ & $219.3 \pm 61.2$ & $\mathrm{H}$ & $29.891 * \star \star$ & $0.000(0.003)$ & 1.000 \\
\hline & & $234.7 \pm 45.5$ & $251.8 \pm 51.0$ & $95.6 \pm 45.8$ & M & 0.850 & $0.357(0.000)$ & 0.152 \\
\hline & $\begin{array}{l}\text { MetS } \\
p \text {-value }\end{array}$ & 0.688 & & & $\mathrm{H} \times \mathrm{M}$ & 1.621 & $0.198(0.000)$ & 0.345 \\
\hline \multirow{3}{*}{$\begin{array}{l}\text { Place } \\
\text { movement }\end{array}$} & \multirow{2}{*}{$\begin{array}{l}\text { Non- } \\
\text { MetS }\end{array}$} & $524.2 \pm 17.6$ & $514.5 \pm 17.6$ & $607.7 \pm 69.5$ & $\mathrm{H}$ & $26.954 * \star *$ & $0.000(0.003)$ & 1.000 \\
\hline & & $474.2 \pm 43.9$ & $490.2 \pm 48.1$ & $344.0 \pm 70.6+\dagger$ & M & $9.888 * *$ & $0.002(0.000)$ & 0.882 \\
\hline & $\begin{array}{l}\text { MetS } \\
p \text {-value }\end{array}$ & 0.253 & & & $\mathrm{H} \times \mathrm{M}$ & $3.559 *$ & $0.028(0.000)$ & 0.663 \\
\hline \multirow{3}{*}{$\begin{array}{l}\text { Recreational } \\
\text { vigorous }\end{array}$} & \multirow{3}{*}{$\begin{array}{l}\text { Non- } \\
\text { MetS } \\
\text { MetS } \\
p \text {-value }\end{array}$} & $212.9 \pm 17.0$ & $209.3 \pm 17.4$ & $243.5 \pm 42.6$ & $\mathrm{H}$ & $30.567 * * *$ & $0.000(0.003)$ & 1.000 \\
\hline & & $191.9 \pm 39.6$ & $194.2 \pm 44.6$ & $172.8 \pm 68.3$ & M & 3.324 & $0.068(0.000)$ & 0.446 \\
\hline & & 0.628 & & & $\mathrm{H} \times \mathrm{M}$ & 0.991 & $0.371(0.000)$ & 0.224 \\
\hline \multirow{3}{*}{$\begin{array}{l}\text { Recreational } \\
\text { moderate }\end{array}$} & \multirow{3}{*}{$\begin{array}{l}\text { Non- } \\
\text { MetS } \\
\text { MetS } \\
p \text {-value }\end{array}$} & $181.8 \pm 9.0$ & $183.0 \pm 9.8$ & $171.1 \pm 22.6$ & $\mathrm{H}$ & $10.371 * \star \star$ & $0.000(0.001)$ & 0.988 \\
\hline & & $149.7 \pm 23.2$ & $147.1 \pm 23.8$ & $171.4 \pm 55.2$ & M & 1.648 & $0.199(0.000)$ & 0.250 \\
\hline & & 0.206 & & & $\mathrm{H} \times \mathrm{M}$ & 0.243 & $0.784(0.000)$ & 0.088 \\
\hline \multirow{3}{*}{$\begin{array}{l}\text { Total physical } \\
\text { activity }\end{array}$} & \multirow{3}{*}{$\begin{array}{l}\text { Non- } \\
\text { MetS } \\
\text { MetS } \\
p \text {-value }\end{array}$} & $1210.2 \pm 48.5$ & $1186.0 \pm 46.6$ & $1417.7 \pm 168.0$ & $\mathrm{H}$ & $64.415^{\star \star \star}$ & $0.000(0.006)$ & 1.000 \\
\hline & & $1182.9 \pm 105.8$ & $1231.8 \pm 116.1$ & $785.4 \pm 172.7+\dagger$ & $M$ & $9.312^{\star *}$ & $0.002(0.000)$ & 0.862 \\
\hline & & 0.812 & & & $\mathrm{H} \times \mathrm{M}$ & $5.062^{\star \star}$ & $0.006(0.000)$ & 0.820 \\
\hline
\end{tabular}

Table 6 shows the differences in energy intake according to the presence or absence of MetS and according to household type. In the total group, young individuals with MetS had significantly higher energy intake than those without MetS (total energy intake and protein intake, $p<0.001$; carbohydrate intake, $p<0.05$ ). For "Total energy intake", "Protein intake", "Fat intake" (all $p<0.001$ ), and for "Carbohydrate intake" ( $p=0.001)$, there was a significant interaction between the presence or absence of MetS and household type. The Bonferroni post-hoc test showed the following group differences: Individuals with MetS living in multi-person households had significantly higher total energy intake, protein intake, fat intake (all $p<0.001$ ), and carbohydrate intake $(p<0.01)$ than those without MetS who lived in multi-person households. Persons with MetS who lived as single persons had significantly lower total 
energy intake $(p<0.05)$ and fat intake $(p<0.01)$ than those without MetS who lived alone. Those with MetS who lived alone had a significantly lower total energy intake and fat intake $(p<0.01)$ than those with MetS who lived in a multi-person household.

Table 6

Energy intake

\begin{tabular}{|c|c|c|c|c|c|c|c|c|}
\hline \multirow[t]{2}{*}{ Factors } & \multirow[t]{2}{*}{ Group } & \multirow[t]{2}{*}{ Total } & \multicolumn{2}{|l|}{ Household type } & \multicolumn{4}{|c|}{ ANOVA } \\
\hline & & & Multiple & Single & F-valu & & $p$-Value $\left(n^{2}\right)$ & Power \\
\hline \multirow{3}{*}{$\begin{array}{l}\text { Total energy } \\
\text { intake (kcal) }\end{array}$} & \multirow{2}{*}{$\begin{array}{l}\text { Non- } \\
\text { MetS }\end{array}$} & $2149.0 \pm 19.4$ & $2137.8 \pm 21.1$ & $2250.1 \pm 56.5$ & $\mathrm{H}$ & $102.233^{* * *}$ & $0.000(0.011)$ & 1.000 \\
\hline & & $2396.7 \pm 58.9$ & $2443.6 \pm 67.9+t+$ & $2012.1 \pm 130.6 † \ddagger$ & M & 0.200 & $0.654(0.000)$ & 0.073 \\
\hline & $\begin{array}{l}\text { MetS } \\
p- \\
\text { value }\end{array}$ & $<0.001$ *** & & & $\mathrm{H} \times \mathrm{M}$ & 28.101 ** & $0.000(0.003)$ & 1.000 \\
\hline \multirow{3}{*}{$\begin{array}{l}\text { Carbohydrate } \\
\text { intake (kacl) }\end{array}$} & \multirow{2}{*}{$\begin{array}{l}\text { Non- } \\
\text { MetS }\end{array}$} & $1173.0 \pm 11.2$ & $1170.8 \pm 12.2$ & $1193.3 \pm 26.9$ & $\mathrm{H}$ & 0.237 & $0.789(0.000)$ & 0.087 \\
\hline & & $1236.5 \pm 24.4$ & $1244.0 \pm 26.2+†$ & $1174.9 \pm 85.8$ & $M$ & 0.252 & $0.616(0.000)$ & 0.079 \\
\hline & $\begin{array}{l}\text { MetS } \\
p- \\
\text { value }\end{array}$ & $0.021 *$ & & & $\mathrm{H} \times \mathrm{M}$ & $7.335^{\star \star}$ & $0.001(0.001)$ & 0.939 \\
\hline \multirow{3}{*}{$\begin{array}{l}\text { Protein } \\
\text { intake } \\
\text { (kcal) }\end{array}$} & \multirow{3}{*}{$\begin{array}{l}\text { Non- } \\
\text { MetS } \\
\text { MetS } \\
p \text { - } \\
\text { value }\end{array}$} & $324.5 \pm 3.8$ & $323.4 \pm 4.2$ & $334.4 \pm 9.2$ & $\mathrm{H}$ & $160.606 * * \star$ & $0.000(0.018)$ & 1.000 \\
\hline & & $362.4 \pm 9.8$ & $367.1 \pm 11.2+† \dagger$ & $323.5 \pm 23.3$ & $M$ & 0.886 & $0.347(0.000)$ & 0.156 \\
\hline & & $<0.001 * \star *$ & & & $\mathrm{H} \times \mathrm{M}$ & 25.2218 *** & $0.000(0.003)$ & 1.000 \\
\hline \multirow{3}{*}{$\begin{array}{l}\text { Fat intake } \\
\text { (kcal) }\end{array}$} & \multirow{3}{*}{$\begin{array}{l}\text { Non- } \\
\text { MetS } \\
\text { MetS } \\
p- \\
\text { value }\end{array}$} & $523.9 \pm 8.8$ & $517.4 \pm 9.2$ & $581.9 \pm 25.2$ & $\mathrm{H}$ & $278.513^{* * \star}$ & $0.000(0.030)$ & 1.000 \\
\hline & & $567.1 \pm 23.1$ & $580.9 \pm 26.6+† \dagger$ & $453.9 \pm 41.2 † \dagger \ddagger$ & $M$ & $7.183^{\star \star}$ & $0.007(0.000)$ & 0.764 \\
\hline & & 0.090 & & & $\mathrm{H} \times \mathrm{M}$ & $30.312^{\star \star \star}$ & $0.000(0.003)$ & 1.000 \\
\hline $\begin{array}{l}\text { Values are exp } \\
\text { syndrome), Int } \\
0.01 \text {, ††+ } p<0 \\
\text { following Bon }\end{array}$ & sed a & $\begin{array}{l}\text { ed with Non } \\
\text { oc test. }\end{array}$ & $\begin{array}{l}\text { errors; MetS, met } \\
\text { Isehold } \times \text { Metabc } \\
\text { etS values, } \ddagger p<c\end{array}$ & $\begin{array}{l}\text { lic syndrome. } \\
\text { syndrome), }{ }^{\star} p \\
\text { compared with }\end{array}$ & $\begin{array}{l}\text { effec } \\
55, * * \\
\text { ultiple }\end{array}$ & $\begin{array}{l}\mathrm{H} \text { (Househol } \\
=0.01, \star \star \star \\
0 \\
\text { alues. Two-w }\end{array}$ & $\begin{array}{l}\text { and } M \text { (Met } \\
.001,+p<0 \text {. } \\
\text { analysis of }\end{array}$ & $\begin{array}{l}\text { lic } \\
\text { †† } p< \\
\text { ance }\end{array}$ \\
\hline
\end{tabular}

\section{Discussion}

This study investigated differences in PA levels and energy intake by household-type and the presence of MetS in a young adult Korean population, to understand the relationships among these factors. We found that components of MetS, such as a large waist circumference, hyperlipidemia, low HDL-C levels, and high fasting blood glucose levels, can be improved by higher PA levels (Very active: $2775.1 \pm 100.2 \mathrm{MET}$ min/week) as compared with inactivity (0-249 MET min/week). Low HDL-C levels could also be improved by increased PA levels (Active: 500-999 MET min/week) as compared with inactivity. In addition, hyperlipidemia, low HDL-C, and high glucose levels can be improved by lower energy intake $(0.8<E E R)$ as compared with higher energy intake (EER > 1.2). Large waist circumference and high glucose were also improved in those with lower energy intake as compared with those with moderate energy intake $(0.8 \leq \mathrm{EER} \leq 1.2)$. In the total group, there was no significant difference between MetS and non-MetS groups in terms of PA levels. This result was similar to that in multi-person households, except for the "Occupational vigorous" category. However, in single-person households, the MetS group had lower levels of "Place movement" and "Total physical activity" than the non-MetS group. Investigating the differences in energy intake according to the presence or absence of MetS and according to household type showed that, in the total group, the MetS group had significantly higher total energy intake, carbohydrate intake, and protein intake levels than the non-MetS group. In multi-person households, the MetS group had significantly higher total energy intake, carbohydrate intake, fat intake, and protein intake than the non-MetS group. However, in single-person households, the MetS group had significantly lower total energy intake and fat intake than the non-MetS group. In 
particular, individuals with MetS who lived in single-person households had lower total energy intake and fat intake than those with MetS who lived in multi-person households. Taken together, our results showed the need for different approaches of implementing PA and nutrition strategies according to household type in order to prevent MetS.

Our results contribute to emerging evidence that PA levels and energy intake are associated with MetS components ${ }^{18,19}$. We found that the prevalence of MetS factors was significantly lower among those with "Very active" PA levels as compared to those with an "Inactive" PA level. Young adults who were very active had a 45\% lower prevalence of MetS. Similar to our study, Sisson et al. ${ }^{20}$ found that men and women with higher levels of sedentary behavior than physical activity in the US had a $66 \%$ higher prevalence of MetS. Those with moderate physical activity (75-180 min/day) had a 29\% lower prevalence of MetS, according to data of 4,327 adults obtained in the NHANEX from 2007 to $2010^{21}$. Moreover, among 410 subjects, aged 18-74 years, subjects with heavy PA levels (based on the Lipid Research Clinics questionnaire) had a $40 \%$ lower prevalence of MetS than subjects with light PA levels ${ }^{22}$. Regular PA has been shown to increase HDL-C in a linear dose--response manner ${ }^{23}$. In addition, intense PA levels could reduce triglyceride levels ${ }^{23}$.

With respect to energy intake, we found that the MetS risk factors had significantly higher values in individuals with moderate or higher energy intake (vs. those with a lower energy intake). Those with a moderate energy intake had a $46 \%$ higher prevalence of having a large waist circumference and a $23 \%$ higher prevalence of having high fasting glucose levels. Moreover, those with a higher energy intake had a 33\% higher prevalence of high triglyceride, $23 \%$ lower prevalence of low LDL-C, and $39 \%$ higher prevalence of high fasting glucose levels. Similar to our study, 7,081 men aged 30 years and older, who overate more than 4 times a week, had a 141\% higher prevalence of MetS ${ }^{24}$. According to the data from the 2007-2012 KNHANES, from 20,515 Korean adults, a high carbohydrate intake (380.8 $\pm 4.7 \mathrm{~g})$ resulted in a $32 \%$ higher prevalence of elevated TG and a $32 \%$ higher prevalence of MetS in men, and a $26 \%$ higher prevalence of elevated TG and $31 \%$ higher prevalence of MetS in women ${ }^{25}$. Moreover, according to a cross-sectional study (6,737 males and 8,845 females) from the 2008-2011 KNHANES, a high carbohydrate intake was associated with a higher prevalence of MetS in males, and a high carbohydrate intake combined with a low fat intake was associated with MetS in females ${ }^{19}$. In addition, according to a study of 3-year KNHANES data, from 2012 to 2014, the prevalence of MetS was positively correlated with carbohydrate intake in an adult population, as assessed by the 24-hour recall questionnaire ${ }^{26}$. These findings confirm that MetS is driven largely by a high nutrient energy intake, particularly that derived from carbohydrates, as young individuals with MetS had a higher carbohydrate intake than those without MetS in this study.

We found that there was no significant difference in PA levels between those with and those without MetS in the total group. However, the MetS group had a higher total energy intake, carbohydrate intake, and protein intake than those without MetS. The prevalence of MetS has increased rapidly in Asia in recent years, and several studies have demonstrated stronger associations between dietary carbohydrate intake and metabolic disease 27,28 . The 2007-2012 National Health and Nutrition Examination Survey studies showed that a high carbohydrate intake is associated with metabolic abnormalities (Ha et al., 2018). According to a cross-sectional study performed in 2018 (6,737 males and 8,845 females), the risk of MetS increased proportionally with the carbohydrate intake proportion ${ }^{25}$. As Korean adults consume more carbohydrates than adults in other regions, stronger associations of dietary carbohydrate with MetS were observed in this study. In addition, studies on the Korean population and a meta-analysis of observational studies have revealed that total, red, and processed meat consumption is associated with a high risk of MetS ${ }^{29}$. Additionally, in a review of epidemiological evidence, dietary protein appeared to increase the risk of MetS ${ }^{30}$. Korean eating habits have changed from a traditional diet focused on vegetables to a western diet focused on meat in recent years. Taken together, MetS in young individuals is more likely to be linked to energy intake than to PA levels.

This study analyzed household-specific PA according to the presence or absence of MetS in young adults. There was no significant difference across PA categories in multi-person households except for the "Occupational vigorous" category. In contrast, place movement and total PA were significantly lower in the MetS group than in the non-MetS group in single-person households. Numerous studies in recent decades have shown that higher PA levels have a favorable impact on each of the MetS components ${ }^{31}$, 32, 33 . In a cross-sectional evaluation of PA and metabolic risk among individuals with a family history of type 2 diabetes, it was suggested that increasing the total amount of PA in sedentary and overweight individuals had beneficial effects on MetS risk ${ }^{34}$. An analysis of MetS in Korean adults, identified MetS risk factors as a lack of walking and flexibility exercises in single-person households ${ }^{35}$. Although living alone does not equate to a lack of a social network or support, social support is important for 
engaging in healthy behaviors, including PA ${ }^{36,37}$. Support from family ${ }^{38}$ and friends ${ }^{39}$ has also been shown to correlate positively with PA. Single-person households without social support could promote a decrease in total PA, which can serve as a potential risk factor for an increased incidence of MetS.

This study also analyzed household-specific dietary intake according to the presence or absence of MetS in young adults. The total energy intake, carbohydrate intake, protein intake, and fat intake were significantly higher in the MetS group than in the non-MetS group in indivuals living in multi-person households. In contrast, the total energy intake and fat intake were significantly lower in the MetS group than in the non-MetS group in individuals living in single-person households. Morever, the total energy intake and fat intake were significantly lower in individuals with MetS who lived alone than in those with MetS who lived in multi-person households. Multi-person households presented a similar tendency to the total group in terms of energy intake: those with MetS tended to have a higher energy intake, as mentioned above, whereas those living alone presented the opposite tendency. According to the 2020 Dietary Reference Intakes for Koreans ${ }^{40}$, the recommended energy intake for men aged 19-49 is about 2,550 calories, and for women aged 19-49, it is about 1,950 calories. The single-person household data in this study represented both men and women. Thus, they consumed relatviely lower amounts of energy than recommended by the guidelines, but were not at a deficient level. Thus, we cautiously speculate that MetS in young adults in single-person households is more likely to be linked to lower PA levels than to energy intake.

MetS is caused by lifestyle factors, such as PA, diet, and weight, and it is reported that the risk of MetS can be reduced by increasing PA levels and by engaging in balanced eating habits $41,42,43$. In this study, we found that there are household-specific aspects to the PA levels and energy intake according to the presence or absence of MetS in young adults. This information can be used as a basis for preparing countermeasures through education on PA and nutrition, and by providing nutrition guidance for young adults with MetS according to the household type. Such practical measures are urgently needed to reduce the incidence of MetS among young Korean adults.

This study's results should be interpreted with consideration of the following limitations. First, we evaluated young adults with MetS, but did not consider the timing of MetS development or the duration of MetS. Second, the amount of PA was not assessed using heart rate measurements or using an accelerometer, but was quantified based on survey findings, which are prone to errors. Third, this study reported simple differences without identifying the causality underlying the relationships between PA and nutrition. Finally, the data generated by using the 24-hour-recall may not represent long-term dietary habits. Twenty-four-hour recall is essentially a retrospective method of diet assessment, where an individual is interviewed about their food and beverage consumption during the previous day or the preceding 24 hours. However, a single 24-hour-recall may not be representative of the habitual diet at an individual level.

\section{Conclusions}

According to the ORs of MetS components, statistically significant associations were observed among low PA levels, high energy intake, and MetS components, including large waist circumference, high TG, low HDL-C, and high fasting glucose levels in young Korean adults. However, our results showed different patterns of associations, according to the household type. In multi-person households, there was no significant difference between individuals with and without MetS, although young adults with MetS had higher energy intake than without MetS. On the other hand, in single-person households, young adults with MetS had lower PA levels and lower energy intake than those without MetS. These findings highlight the need for different approaches of implementing PA and nutrition strategies according to the household type, in order to prevent MetS. Programs aimed at decreasing energy intake are necessary for multi-person households, while programs aimed at increasing PA levels and fat intake and balanced nutrition are needed for single-person households. Current trends to use personal devices, smartphones, activity trackers to measure physical activity, and applications to measure energy intake can provide innovative opportunities for researchers and healthcare providers to educate clients and intervene in new ways to promote healthy lifestyles.

\section{Declarations}

\section{Acknowledgments:}

Page $12 / 16$ 
This study analyzed the data provided by the Korean National Health and Nutrition Examination Survey 2016-2018, conducted by the Korea Centers for Disease Control and Prevention.

\section{Author Contributions:}

Conception and study design, Y.L., J.L., and J.P.; Statistical Analysis, Y.L., Y.P., and J.P.; Investigation, Y.L., and E.S., and Data Interpretation Y.L., and J.P.; Writing-Original Draft Preparation, Y.L., and J.P.; Writing-Review \& Editing, Y.L., E.S., H.L., and J.P.; Supervision, J.P. All authors have read and approved the final manuscript.

\section{Funding:}

No specific funding was received for this study.

\section{Availability of data and materials:}

The dataset can be downloaded from Korea National Health and Nutrition Examination Survey website (https://www.kdca.go.kr/index.es?sid=a3)

\section{Ethics approval and consent to participate:}

The KNHANES consists of a health interview survey, a nutrition survey, and a health examination, and is conducted according to the Declaration of Helsinki. This survey was approved by the Institutional Review Board of the Korea Centers for Disease Control and Prevention (reference number: 2018-01-03-P-A). All participants in the survey signed an informed consent form.

\section{Consent for publication:}

Not applicable.

\section{Competing interests:}

The authors declare no conflict of interest.

\section{References}

1. Yeung, W. J. J., \& Cheung, A. K. L. (2015). Living alone: One-person households in Asia. Demographic Research, 32(1), 10991112. https://doi.org/10.4054/DemRes.2015.32.40

2. Cho, Young Kyung, Kyung Won Shim, Hye Won Suk, Hong Soo Lee, Sang Wha Lee, A Ri Byun, H. N. L. (2019). Differences between One-Person and Multi-Person Households on Socioeconomic Status, Health Behavior, and Metabolic Syndrome Across Gender and Age Groups. Korean Journal of Family Practice, 9(4), 373-382.

3. Korean Statistical Information Service. (2021). Census. https://kosis.kr/statisticsList/statisticsListlndex.do? publication $Y N=Y \&$ statld=1962001\#content-group

4. Lim, S., Shin, H., Song, J. H., Kwak, S. H., Kang, S. M., Yoon, J. W., Choi, S. H., Cho, S. II, Park, K. S., Lee, H. K., Jang, H. C., \& Koh, K. K. (2011). Increasing prevalence of metabolic syndrome in Korea: The Korean National Health and Nutrition Examination Survey for 1998-2007. Diabetes Care, 34(6), 1323-1328. https://doi.org/10.2337/dc10-2109

5. Kim, S., Lee, K., \& Lee, Y. (2018). Selection attributes of home meal replacement by food-related lifestyles of single-person households in South Korea. Food Quality and Preference, 66(December 2017), 44-51.

https://doi.org/10.1016/j.foodqual.2018.01.004

6. Sui, X., LaMonte, M. J., Laditka, J. N., Hardin, J. W., Chase, N., Hooker, S. P., \& Blair, S. N. (2007). Cardiorespiratory fitness and adiposity as mortality predictors in older adults. Journal of the American Medical Association, 298(21), 2507-2516. https://doi.org/10.1001/jama.298.21.2507

7. Tran, B. T., Jeong, B. Y., \& Oh, J. K. (2017). The prevalence trend of metabolic syndrome and its components and risk factors in Korean adults: results from the Korean National Health and Nutrition Examination Survey 2008-2013. BMC Public Health, 17(1), 1-8. https://doi.org/10.1186/s12889-016-3936-6

Page $13 / 16$ 
8. Hetherington, M. M., Anderson, A. S., Norton, G. N. M., \& Newson, L. (2006). Situational effects on meal intake: A comparison of eating alone and eating with others. Physiology and Behavior, 88(4-5), 498-505.

https://doi.org/10.1016/j.physbeh.2006.04.025

9. Kim, C. K., Kim, H. J., Chung, H. K., \& Shin, D. (2018). Eating alone is differentially associated with the risk of metabolic syndrome in Korean men and women. International Journal of Environmental Research and Public Health, 15(5), 1-14. https://doi.org/10.3390/ijerph15051020

10. Kwon, A. R., Yoon, Y. S., Min, K. P., Lee, Y. K., \& Jeon, J. H. (2018). Eating alone and metabolic syndrome: A population-based Korean National Health and Nutrition Examination Survey 2013-2014. Obesity Research and Clinical Practice, 12(2), 146-157. https://doi.org/10.1016/j.orcp.2017.09.002

11. Lee, S. J., \& Lee, S. H. (2021). Comparative Analysis of Health Behaviors, Health Status, and Medical Needs among One-Person and MultiPerson Household Groups: Focused on the Ageing Population of 60 or More. Korean Journal of Family Medicine, 42(1), 73-83. https://doi.org/10.4082/kjfm.20.0014

12. Choi, S., Kwak, J. H., Chung, H. K., Kang, H. J., \& Paik, J. K. (2020). Comparison of Nutrient Intake and Metabolic Syndrome between Single Person Households and Non-Single Person Households in Elderly Subjects - From the Sixth Korea National Health and Nutrition Examination Survey (KNHANES, 2013 2015) -. Korean Journal of Food Nutrition, 33(3), 322-330.

13. Oh, S. W. (2011). Obesity and Metabolic Syndrome in Korea. Diabetes and Metabolism Journal, 35, 561-566. https://doi.org/10.1016/S1499-2671(11)80002-X

14. Circulation. (2002). Third report of the National Cholesterol Education Program (NCEP) Expert Panel on Detection, Evaluation, and Treatment of High Blood Cholesterol in Adults (Adult Treatment Panel III) Final report. In Circulation (Vol. 106).

15. Grundy, S. M., Cleeman, J. I., Daniels, S. R., Donato, K. A., Eckel, R. H., Franklin, B. A., Gordon, D. J., Krauss, R. M., Savage, P. J., Smith, S. C., Spertus, J. A., \& Costa, F. (2005). Diagnosis and management of the metabolic syndrome: An American Heart Association/National Heart, Lung, and Blood Institute scientific statement. Circulation, 112(17), 2735-2752. https://doi.org/10.1161/CIRCULATIONAHA.105.169404

16. Seo, M. H., Lee, W. Y., Kim, S. S., Kang, J. H., Kang, J. H., Kim, K. K., Kim, B. Y., Kim, Y. H., Kim, W. J., Kim, E. M., Kim, H. S., Shin, Y. A., Shin, H. J., Lee, K. R., Lee, K. Y., Lee, S. Y., Lee, S. K., Lee, J. H., Lee, C. B., ... Yoo, S. J. (2019). 2018 Korean society for the study of obesity guideline for the management of obesity in Korea. Journal of Obesity and Metabolic Syndrome, 28(1), 40-45. https://doi.org/10.7570/JOMES.2019.28.1.40

17. WHO. (2012). Global Physical Activity Questionnaire (GPAQ) Analysis Guide. In Geneva: World Health Organization. http://scholar.google.com/scholar?hl=en\&btnG=Search\&q=intitle:Global+Physical+Activity+Questionnaire+ (GPAQ)+Analysis+Guide\#1

18. He, D., Xi, B., Xue, J., Huai, P., Zhang, M., \& Li, J. (2014). Association between leisure time physical activity and metabolic syndrome: A meta-analysis of prospective cohort studies. Endocrine, 46(2), 231-240. https://doi.org/10.1007/s12020-0130110-0

19. Kwon, Y. J., Lee, H. S., \& Lee, J. W. (2018). Association of carbohydrate and fat intake with metabolic syndrome. Clinical Nutrition, 37(2), 746-751. https://doi.org/10.1016/j.clnu.2017.06.022

20. Sisson, S. B., Camhi, S. M., Church, T. S., Martin, C. K., Tudor-Locke, C., Bouchard, C., Earnest, C. P., Smith, S. R., Newton, R. L., Rankinen, T., \& Katzmarzyk, P. T. (2009). Leisure time sedentary behavior, occupational/domestic physical activity, and metabolic syndrome in U.S. men and women. Metabolic Syndrome and Related Disorders, 7(6), 529-536. https://doi.org/10.1089/met.2009.0023

21. Frugé, A. D., Byrd, S. H., Fountain, B. J., Cossman, J. S., Schilling, M. W., \& Gerard, P. (2015). Increased physical activity may be more protective for metabolic syndrome than reduced caloric intake. An analysis of estimated energy balance in U.S. adults: 2007-2010 NHANES. Nutrition, Metabolism and Cardiovascular Diseases, 25(6), 535-540. https://doi.org/10.1016/j.numecd.2015.03.006

22. Mirmiran, P., Noori, N., \& Azizi, F. (2008). A prospective study of determinants of the metabolic syndrome in adults. Nutrition, Metabolism and Cardiovascular Diseases, 18(8), 567-573. https://doi.org/10.1016/j.numecd.2007.06.002

23. Mann, S., Beedie, C., \& Jimenez, A. (2014). Differential effects of aerobic exercise, resistance training and combined exercise modalities on cholesterol and the lipid profile: review, synthesis and recommendations. Sports Medicine, 44(2), 211-221. 
https://doi.org/10.1007/s40279-013-0110-5

24. Shin, A., Lim, S. Y., Sung, J., Shin, H. R., \& Kim, J. (2009). Dietary Intake, Eating Habits, and Metabolic Syndrome in Korean Men. Journal of the American Dietetic Association, 109(4), 633-640. https://doi.org/10.1016/j.jada.2008.12.015

25. Ha, K., Kim, K., Chun, O. K., Joung, H., \& Song, Y. (2018). Differential association of dietary carbohydrate intake with metabolic syndrome in the US and Korean adults: Data from the 2007-2012 NHANES and KNHANES. European Journal of Clinical Nutrition, 72(6), 848-860. https://doi.org/10.1038/s41430-017-0031-8

26. Ahn, J., Kim, N. S., Lee, B. K., \& Park, S. (2017). Carbohydrate intake exhibited a positive association with the risk of metabolic syndrome in both semi-quantitative food frequency questionnaires and 24-hour recall in women. Journal of Korean Medical Science, 32(9), 1474-1483. https://doi.org/10.3346/jkms.2017.32.9.1474

27. McKeown, N. M., Meigs, J. B., Liu, S., Saltzman, E., Wilson, P. W. F., \& Jacques, P. F. (2004). Carbohydrate Nutrition, Insulin Resistance, and the Prevalence of the Metabolic Syndrome in the Framingham Offspring Cohort. Diabetes Care, 27(2), 538546. https://doi.org/10.2337/diacare.27.2.538

28. Finley, C. E., Barlow, C. E., Halton, T. L., \& Haskell, W. L. (2010). Glycemic index, glycemic load, and prevalence of the metabolic syndrome in the cooper center longitudinal study. Journal of the American Dietetic Association, 110(12), 1820-1829. https://doi.org/10.1016/j.jada.2010.09.016

29. Kim, Y., \& Je, Y. (2018). Meat consumption and risk of metabolic syndrome: Results from the Korean population and a metaanalysis of observational studies. Nutrients, 10(4). https://doi.org/10.3390/nu10040390

30. Baxter, A. J., Coyne, T., \& McClintock, C. (2006). Dietary patterns and metabolic syndrome - A review of epidemiologic evidence. Asia Pacific Journal of Clinical Nutrition, 15(2), 134-142.

31. Church, T. (2011). Exercise in Obesity, Metabolic Syndrome, and Diabetes. Progress in Cardiovascular Diseases, 53(6), 412418. https://doi.org/10.1016/j.pcad.2011.03.013

32. Zhang, D., Liu, X., Liu, Y., Sun, X., Wang, B., Ren, Y., Zhao, Y., Zhou, J., Han, C., Yin, L., Zhao, J., Shi, Y., Zhang, M., \& Hu, D. (2017). Leisure-time physical activity and incident metabolic syndrome: a systematic review and dose-response meta-analysis of cohort studies. Metabolism: Clinical and Experimental, 75, 36-44. https://doi.org/10.1016/j.metabol.2017.08.001

33. Strasser, B. (2013). Physical activity in obesity and metabolic syndrome. Annals of the New York Academy of Sciences, 1281(1), 141-159. https://doi.org/10.1111/j.1749-6632.2012.06785.x

34. Ekelund, U., Griffin, S. J., \& Wareham, N. J. (2007). Physical activity and metabolic risk in individuals with a family history of type 2 diabetes. Diabetes Care, 30(2), 337-342. https://doi.org/10.2337/dc06-1883

35. An, B., \& Son, J. (2018). Analysis of Metabolic Syndrome in Korean Adult One-Person Households. Journal of Korean Public Health Nursing, August 2017, 30-43.

36. Boslaugh, S. E., \& Andresen, E. M. (2006). Correlates of physical activity for adults with disability. Preventing Chronic Disease, $3(3), 1-14$.

37. Austin, S., Qu, H., \& Shewchuk, R. M. (2013). Health care providers' recommendations for physical activity and adherence to physical activity guidelines among adults with arthritis. Preventing Chronic Disease, 10(11), 1-10.

https://doi.org/10.5888/pcd10.130077

38. Lindsay Smith, G., Banting, L., Eime, R., O’Sullivan, G., \& van Uffelen, J. G. Z. (2017). The association between social support and physical activity in older adults: A systematic review. International Journal of Behavioral Nutrition and Physical Activity, 14(1), 1-21. https://doi.org/10.1186/s12966-017-0509-8

39. Sarkar, S., Taylor, W. C., Lai, D., Shegog, R., \& Paxton, R. J. (2016). Social support for physical activity: Comparison of family, friends, and coworkers. Work, 55(4), 893-899. https://doi.org/10.3233/WOR-162459

40. Ministry of Health and Welfare, \& The Korean Nutrition Society. (2020). 2020 Dietary Reference Intakes for Korean: Energy and Macronutrients.

41. Serrano-Sánchez, J. A., Fernández-Rodríguez, M. J., Sanchis-Moysi, J., del Cristo Rodríguez-Pérez, M., Marcelino-Rodríguez, l., \& de León, A. C. (2019). Domain and intensity of physical activity are associated with metabolic syndrome: A population-based study. PLOS ONE, 14(7), 1-17. https://doi.org/10.1371/journal.pone.0219798

42. Kim, Y. J., Hwang, J. Y., Kim, H., Park, S., \& Kwon, O. (2019). Diet quality, physical activity, and their association with metabolic syndrome in Korean adults. Nutrition, 59, 138-144. https://doi.org/10.1016/j.nut.2018.08.009

Page $15 / 16$ 
43. Jung, W.-S., Hwang, H., Kim, J., Park, H.-Y., \& Lim, K. (2019). Effect of interval exercise versus continuous exercise on excess post-exercise oxygen consumption during energy-homogenized exercise on a cycle ergometer. Journal of Exercise Nutrition \& Biochemistry, 23(2), 45-50. https://doi.org/10.20463/jenb.2019.0016

\section{Figures}

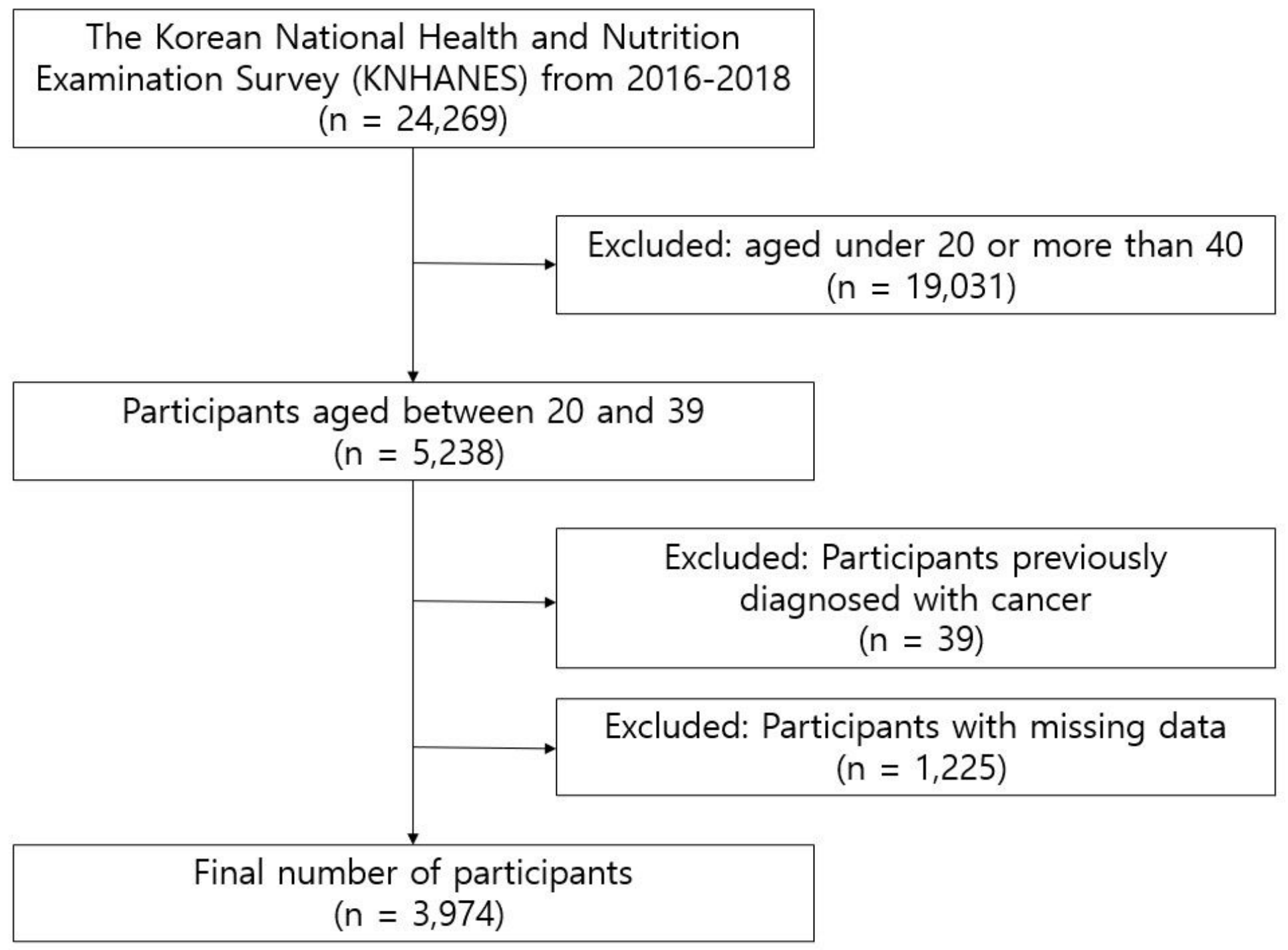

Figure 1

Flow diagram for the selection of study participants 